Daniela Gennari Pires de Toledo

Revisão taxonômica do grupo de tríades pertencente ao gênero Oxyrhopus (Serpentes:

Pseudoboini)

Taxonomic review of the triad group

belonging to the genus Oxyrhopus (Serpentes:

Pseudoboini) 
Daniela Gennari Pires de Toledo

\title{
Revisão taxonômica do grupo de tríades pertencente ao gênero Oxyrhopus (Serpentes: Pseudoboini)
}

\author{
Taxonomic review of the triad group \\ belonging to the genus Oxyrhopus (Serpentes: \\ Pseudoboini)
}

Dissertação apresentada ao Instituto de Biociências da Universidade de São Paulo, para a obtenção de Título de Mestre em Zoologia, na Área de Taxonomia.

Orientador: Hussam El Dine Zaher

São Paulo 
Gennari P. T., Daniela

Revisão taxonômica do grupo de tríades pertencente ao gênero Oxyrhopus (Serpentes: Pseudoboini)

130 páginas

Dissertação (Mestrado) - Instituto de Biociências da Universidade de São Paulo. Departamento de Zoologia

1. Taxonomia 2. Xenodontinae 3.

Morfologia 4. Falsa-coral Universidade de São Paulo. Instituto de Biociências.

Departamento de Zoologia.

Comissão Julgadora:

$\operatorname{Prof}(a) . \operatorname{Dr}(a)$.

$\operatorname{Prof}(a) . \operatorname{Dr}(a)$.

Prof. Dr. Hussam El Dine Zaher

Orientador 
DEDICATÓRIA

Aos meus avós, Florinda e Pedro

Gennari, Ana e Archangelo Pires de Toledo, pela vontade de tê-los aqui pertinho para compartilhar comigo esse momento. Saudades! 
"Sigo convicta, na tentativa de manter a calma Mesmo estando no olho do furacão Mesmo quando o coração aperta Mesmo com o planeta em estado de alerta Seguindo, no desafio de manter a mente quieta, a espinha ereta, e a cabeça no lugar. Na medida do possível, na sintonia certa..." 


\section{AGRADECIMENTOS}

Então, vamos lá! Finito. Depois de dois anos (e meio) de muito aprendizado, amadurecimento pessoal e profissional, perrengues e prestígios, encerro aqui hoje uma etapa importante da minha formação. E nada disso seria possível se não tivesse tido ao meu lado boas companhias, boas influências e boa energias. Acho difícil lembrar de todos os nomes que contribuíram de alguma forma comigo nessa caminhada, portanto fica aqui registrado minha enorme gratidão à todxs elxs.

Agradeço ao meu orientador, prof. Hussam Zaher, primeiro pela oportunidade de trabalhar anteriormente na parte técnica e curatorial dessa imensa e fascinante Coleção Herpetológica do MZUSP. A partir daí, por me incentivar à academia e permitir desenvolver o projeto que me instigava desde a iniciação. Agradeço pela amizade e pela confiança depositada em mim e no meu trabalho, pelos ensinamentos, pelas conversas formais e informais que me fizeram amadurecer um bocado ao longo desses anos.

Agradeço ao mestre Valdir José Germano, que de perto ou de longe tem papel fundamental na minha formação há mais de dez anos. Tenho por ti gratidão e admiração imensa Val, pela pessoa que você é, pelas valiosas aulas diárias na saudosa coleção do Buta. Pelo apoio de sempre e por acreditar em mim, ouvir minhas indagações e me acalmar sempre com palavras positivas. Amo você, nego!

Agradeço também ao cara que tem um coração enorme e me abriu as portas à Herpetologia em 2008, Francisco Luís Franco, e me orientou nos meus primeiros passos com as Oxyrhopus. Kikão, obrigada por tudo, parceiro!

Aos membros da banca, os quais tenho grande admiração, sou grata por aceitarem participar desse momento. Tenho a certeza que seus comentários, correções, críticas e sugestões irão enriquecer significativamente o trabalho, visto que já aprendi bastante com cada um de vocês ao longo dessa caminhada. Aninha e Felipão, Giovanna e Fausto, meu muito obrigada! 
Ao amigo Juan Camilo Arredondo, por toda a força principalmente nessa reta final, pela ajuda com as análises, pelas horas de Skype (manhã, tarde, noite e madrugadas), por aguentar minhas euforias pelas discussões e conversas sempre produtivas. Também agradeço por me ceder as imagens e dados dos museus aos quais não pude visitar (ICN, MUSM, CORBIDI e USNM). Juan, você foi parte fundamental nesse trabalho.

Ao amigo Felipe Grazziotin, pelo apoio de sempre, profissional e pessoal. Pelos campos, pelas conversas, pelas cervejas, pelo incentivo e pela confiança. Admiro você pela pessoa e pelo profissional que você é.

Sou profundamente grata a todxs xs curadorxs que me disponibilizaram o material para que esse trabalho fosse concluído. Desta forma, agradeço à prof. Dra. Ana Prudente (MPEG), ao prof. Dr. Felipe Curcio (UFMT), ao prof. Dr. Paulo Passos (UFRJ), Ao prof. Dr. Antonio Argôlo (MZUESC e CZGB) e prof. Dr. Felipe Grazziotin (IBSP) pela recepção e acolhimento nas coleções. A prof. Dra. Fernanda Werneck e Ariane Silva (INPA), prof. Dr. Pedro Nunes (UFPE), prof. Dr. Daniel Mesquita e Fagner Delfim (UFPB), prof. Dr. Paulo Garcia e Camila Almeida (UFMG) pelo empenho no envio do material solicitado.

Ao Museu de Zoologia da USP, minha segunda casa nesses dois anos, serei eternamente grata ao acolhimento e as amizades que fiz aqui. Quero agradecer aos amigos que já não estão mais no laboratório mas foram fundamentais na minha chegada: Ambrosina Tomas, Felipe Grazziotin, Beta Graboski, Giovanna Montingelli, Vivian Trevine, Fausto Barbo (vai curin!), Ana Botallo, Juan Pablo Hurtado, Hana Suzuki, Juan Camilo Arredondo, Ricardo Fuentes, Paola Sanchés, Léo Malagoli, Léo Oliveira, Gabriela Sobral Flavio Molina e Gabriela Sanches. Aos amigos que ainda continuam, Alberto Carvalho, Bruno Navarro, Luis Bio, Rodolfo Santos, Lucas Piazentin, Paulo Machado, Bruno Augusta André Braga e Francisco Brum.

Aos estagiários que nos acompanham atualmente, agradeço o empenho na execução das atividades rotineiras. É lindo ver o brilho no olhar de vocês ao trabalhar com os bichos. Me traz à tona a menina que entrou no Butantan em 2008 e quase caiu de costas 
ao ver a coleção. Avante! Tenho que destacar entre esses, Luana Genter. Lu, muito obrigada pela força com os bichos no museu. Sua ajuda certamente enriqueceu esse trabalho.

Além disso, agradeço ao pessoal do futebol de quinta do MZUSP, que tornaram minhas semanas mais leves nesse último ano.

Agradeço ao amigo que além do laboratório dividiu a casa comigo e trouxe toda sua malemolência cubana para alegrar os nossos dias, Ernesto Aranda. Saudades de ti.

Um agradecimento especial vai para o time que se formou nesse tempo de trabalho. Às minhas amoras, Natália Rizzo Friol, Daniella França e Aline Staskowian Benetti, minha eterna gratidão por todos os momentos de descontração no laboratório e fora dele. Mas também por todo apoio, pelos conselhos e paciência nos momentos de tensão e desespero. Amo vocês!

Aos amigos do coração, Bruno Rocha, Livia Corrêa, Frederico Alcântara, Flora Ortiz, Jade Lima e Betina Chaluppe. Quero vocês comigo, sempre.

À Laura Alencar, pela amizade de uma década, pelos conselhos e puxões de orelha, pelas risadas e longas conversas sobre os mais diversos assuntos.

Aos meus pais, Lia Gennari e Valencio Toledo, minhas irmãs Renata e Adriana e os "cunhas" Léo e Héio, pelo apoio e incentivo nesses anos. Pelo amor e acolhimento em todos os momentos. Vocês são meu porto seguro, minha base, que eu amo incondicionalmente. Tamo junto!!!

À toda minha família, as Gennaris e os Toledos, pelos encontros e pelas festas que tornam a vida mais divertida. $E$ à minha mais nova família que me acolheu de uma forma inexplicável, quero agradecer aos Fiordoliva por todo amor e carinho que recebi.

E à Maria Cecília Fiordoliva, minha Ceci, quero agradecer de todo meu coração a força, o apoio e a paciência que teve comigo dia a dia nessa reta final. Agradeço pelo encontro inesperado que essa vida nos trouxe e pelo amor que construímos juntas. Quero compartilhar contigo o que há de melhor e mais gostoso na vida. Te amo! 


\section{INTRODUÇÃO}

A subordem Serpentes Linnaeus, 1758 possui atualmente 3709 espécies descritas (Uetz, 2019), divididas em dois clados distintos: Scolecophidia e Alethinophidia. Este último é composto pela maior diversidade de espécies viventes, incluindo as serpentes avançadas do grupo Caenophidia (Zaher et al., 2009; 2019).

Há décadas os Caenophidia têm sido alvo de diversos debates acerca das relações filogenéticas de seus integrantes (Zaher et al., 2009; Oguiura et al., 2009; Vidal et al., 2010; Grazziotin et al., 2012; Pyron et al., 2011; 2013; Figueiroa et al., 2016), estabelecendo nesse cenário um período de instabilidade taxonômica, com esquemas de classificação divergentes e as vezes contraditórios (Zaher et al., 2019).

Recentemente, um estudo utilizou evidências moleculares e morfológicas de crânio, vértebras e caracteres hemipenianos dos Caenophidia, combinando também informações do registro fóssil em uma árvore calibrada no tempo, visando elucidar os padrões atuais de diversificação das serpentes avançadas nos níveis de família e também suprafamiliares (Zaher et al., 2019). Neste mesmo estudo, em análise que abrange $78 \%$ dos gêneros pertencentes à família Dipsadidae Bonaparte 1838, a mesma foi recuperada como um clado robusto apresentando uma dicotomia basal entre o gênero Asiático Thermophis e um clado fortemente suportado, que inclui todos os dipsadídeos do Novo Mundo (veja também He et al., 2009).

A linhagem de Dipsadidae do Novo Mundo é considerada uma das

maiores irradiações dentre as serpentes Caenophidia. Atualmente, a família é composta por 96 gêneros e 764 espécies distribuídas nas Américas e nas Índias Ocidentais que, por sua vez, encontram-se divididas 
em três subfamílias: Dipsadinae, Carphophiinae e Xenodontinae, além de outros táxons ainda considerados como incertae sedis (Zaher et al., 2019).

Dentre as três subfamílias reconhecidas, Xenodontinae apresenta-se como a mais diversa, incluindo 342 espécies distribuídas em 15 tribos: Alsophiini, Caaeteboiini, Conophiini, Echinantherini, Elapomorphini, Eutrachelophiini, Hydrodynastini, Hydropsini, Pseudoboini, Psomophiini, Philodryadini, Saphenophiini, Tachymenini, Tropidrodryadini e Xenodontini.

As serpentes da tribo Pseudoboini compõem, atualmente, 11 gêneros distribuídos desde a região sul do México até a Argentina (Jenner \& Dowling, 1985; Zaher, 1994; Grazziotin et al., 2012). São eles: Boiruna Zaher, 1996; Clelia Fitzinger, 1826; Drepanoides Dunn, 1928; Mussurana Zaher et al 2009; Oxyrhopus Wagler, 1830 Paraphimophis Grazziotin et al 2012; Phimophis Cope, 1860; Pseudoboa Schneider, 1801; Rhachidelus Boulenger, 1908 Rodriguesophis Grazziotin et al 2012 e Siphlophis Fitzinger, 1843.

Seus representantes são caracterizados por compartilhar uma série de estruturas morfológicas externas, ósseas e hemipenianas (Bailey, 1967; Zaher, 1994; 1999), além de apresentar alto suporte molecular desde estudos pioneiros na área (Cadle 1984; Vidal et al., 2000; Zaher et al., 2009; Vidal et al., 2010; Grazziotin et al., 2012). Sendo assim, a tribo é reconhecida atualmente como um agrupamento monofilético, sustentada por caracteres morfológicos e moleculares. Zaher (1999) aponta para os problemas na taxonomia dos Pseudoboini e sugere que importantes mudanças taxonômicas devem ser feitas a fim de melhor representar a filogenia da tribo. Ainda hoje, persistem questões não esclarecidas a respeito do posicionamento filogenético e relação de parentesco dos gêneros de pseudoboíneos. Entretanto, sabe-se que o gênero Oxyrhopus 
forma um grupo historicamente relacionado aos gêneros Clelia e Pseudoboa (Cunha \& Nascimento, 1983; Bernardo, 2010).

Ainda no século XIX, na tentativa de classificar suas espécies, os táxons reconhecidos como pertencentes ao gênero Oxyrhopus foram alocados por diversas vezes nos gêneros acima citados e, ainda, em outros gêneros, como Sphenocephalus Fitzinger, 1843, gênero no qual foi descrito O. melanogenys (Tschudi, 1845) - uma das espécies alvo do presente estudo - sendo também, posteriormente, citada como Tachymenis bitorquata por Gunther (1872). Boulenger (1896) fundiu sob a designação de Oxyrhopus os gêneros Pseudoboa e Clelia por não reconhecer diferenças suficientes entre os táxons. Stejneger (1901) separou novamente os três gêneros e incluiu dentro do gênero Pseudoboa apenas as espécies com subcaudais inteiras. Já as demais espécies, que apresentam subcaudais divididas, foram alocadas no gênero Clelia, que tem prioridade nomenclatural sobre Oxyrhopus. Gomes (1918) discordando desta posição, reuniu os três gêneros novamente, porém sob o nome Pseudoboa, que tem prioridade sobre Oxyrhopus e Clelia, proposta que foi seguida por Amaral (1926). Desta forma, a classificação taxonômica das espécies permaneceu confusa até meados do século XX.

Ao estudar os ofídios colombianos, Dunn (1944) elaborou uma chave artificial para os gêneros reconhecidos no país, dividindo-os em três grandes grupos e diagnosticando Oxyrhopus, Pseudoboa e Clelia. Roze (1966) acompanhou essa separação para as Oxyrhopus e Pseudoboa da Venezuela, utilizando as diferenças no número de escamas dorsais e subcaudais já citadas anteriormente na literatura e acrescentando caracteres como a dentição e conformação dos hemipênis. Por fim, Bailey (1970) caracterizou mais nitidamente os gêneros, com chaves de identificação elucidativas, reconhecendo para Oxyrhopus cinco grupos de 
espécies e subespécies. Entre estes, Bailey reuniu as espécies que apresentavam coloração em tríades no grupo melanogenys.

O gênero Oxyrhopus contém atualmente 14 espécies válidas, distribuídas por toda a América Latina, sendo estas: Oxyrhopus clathratus Duméril, Bibron \& Duméril, 1854; Oxyrhopus doliatus Duméril, Bibron \& Duméril, 1854; Oxyrhopus erdisii (Barbour, 1913); Oxyrhopus fitzingeri (Tschudi, 1845); Oxyrhopus formosus (Wied-Neuwied, 1820); Oxyrhopus guibei Hoge \& Romano, 1977; Oxyrhopus leucomelas (Werner, 1916); Oxyrhopus marcapatae (Boulenger, 1902); Oxyrhopus melanogenys (Tschudi, 1845); Oxyrhopus occipitalis (Wied-Neuwied, 1824); Oxyrhopus petolarius (Linnaeus, 1758); Oxyrhopus rhombifer Duméril, Bibron \& Duméril, 1854; Oxyrhopus trigeminus Duméril, Bibron \& Duméril, 1854; Oxyrhopus vanidicus Lynch, 2009.

As espécies de Oxyrhopus possuem porte médio, dentição opistóglifa, reprodução ovípara, pupilas elípticas e padrão coral de coloração. Além disso, alimentam-se basicamente de lagartos e pequenos mamíferos e possuem hábito predominantemente noturno (Bailey, 1967; Pizzatto \& Marques, 2002; Alencar et al., 2013; Gaiarsa et al., 2013).

Dentre as espécies reconhecidas do gênero, Oxyrhopus rhombifer, O. clathratus, O. petola, O. doliatus, O. erdisii, O. fitzingeri, O. marcapatae e O. leucomelas caracterizam-se por apresentar padrão de desenho variegado ou com bandas simples, em mônades ou losangulares no dorso (Argolo, 2004; Campbell \& Lamar, 1989; Freitas \& Silva, 2007; Giraudo, 2001; Kornacker, 1999; Perez-Santos \& Moreno, 1988; Bernardo, 2010). Oxyrhopus formosus e O. occipitalis apresentam o dorso vermelho ou alaranjado uniforme e a região rostral e internasal comumente amarelas (Campbell \& Lamar, 1989; Mcculloch, et al 2009). As demais espécies possuem padrão dorsal de cor em tríades separadas por bandas 
que variam do vermelho escuro ao amarelo, e tratam-se das espécies alvo deste estudo.

Lynch (2009), em seu estudo sobre taxonomia e variação geográfica das espécies de Oxyrhopus da Colômbia, descreveu O. vanidicus a partir de 44 espécimes previamente classificados como $O$. melanogenys (e $O$. aff melanogenys). $\mathrm{O}$ autor utilizou como diagnose o padrão de cor em tríades, a fusão das tríades anteriores e a ausência de um par de bandas escuras e estreitas na nuca. Foi especificado pelo autor que as bandas laranjas na região anterior do corpo são estreitas e muitas vezes não formam bandas completas e, já na parte posterior, tornam-se progressivamente maiores formando anéis completos. Não há, desde então, estudo que procure abranger toda a distribuição da espécie, tampouco uma descrição da variação morfológica externa e hemipeniana.

Oxyrhopus melanogenys (Tschudi 1845) foi descrita originalmente como Sphenocephalus melanogenys, sem a determinação de holótipo e localidade-tipo. Em uma breve descrição em latim (Figura 1), Tschudi relatou que a nova espécie apresenta a parte anterior vermelha pintada com bandas transversais pretas que desaparecem no fim do corpo e ventre com pontos pretos na parte posterior. Um ano depois, em um extenso trabalho sobre a Fauna Peruana, o mesmo autor descreveu de forma mais detalhada e ilustrou (Figura 2) S. melanogenys (Tschudi, 1846). Posteriormente, Gunther (1872) descreveu Tachymenis bitorquata para a Amazônia Peruana, como espécie que possui dois colares pretos na nuca, corpo e ventre amarelo. Boulenger (1896), em seu catálogo de serpentes do museu de história natural britânico, reconheceu tanto Oxyrhopus melanogenys como O. bitorquatus ilustrando apenas essa última (Figura 3). Em sua descrição, Boulenger caracterizou $O$. melanogenys como tendo apenas a parte anterior do corpo ornada por tríades de faixas pretas transversais e um ventre amarelo. Em sua chave taxonômica das espécies de serpentes 
neotropicais, Bailey (1970) considerou O. bitorquatus como sendo um sinônimo junior de $O$. melanogenys Tschudi 1846. Até o presente momento, a distribuição da espécie ocorre ao longo da Amazônia brasileira e no Peru ocidental e, atualmente, há duas subespécies reconhecidas: Oxyrhopus melanogenys melanogenys e O. m. orientalis Cunha \& Nascimento 1983. Em uma revisão das espécies dos gêneros Oxyrhopus e Pseudoboa de ocorrência na Amazônia oriental (Amapá, Pará) e Maranhão, Cunha \& Nascimento descreveram O. m. orientalis, como subespécie caracterizada pelo padrão cromático, constituído de bandas negras estreitas, dispostas em tríades, com duas bandas laterais muito estreitas e uma mais larga central, cada uma separada por uma estreita banda vermelha. Os autores referem-se à população que abrange a porção mais oriental da Amazônia - anteriormente determinada pelos próprios como O. trigeminus incorretamente Cunha (\& Nascimento, 1978) - e isolada de $O$. m. melanogenys por longo hiato geográfico.

Oxyrhopus trigeminus Duméril, Bibron \& Duméril, 1854 foi descrita com base em cerca de 30 espécimes provenientes da Bahia e do Rio de Janeiro (Brasil), sem a designação de um holótipo. Vanzolini (1948) restringiu a localidade-tipo para o Distrito Federal, Brasil (= Rio de Janeiro). Esta proposta não foi aceita por Bailey in Peters \& OrejasMiranda (1970), postura com a qual Zaher \& Caramaschi (1992) concordaram. Desta forma, no artigo revisivo de Zaher \& Caramaschi (1992), o espécime MNHN 7599 procedente do estado da Bahia, foi designado como lectótipo de $O$. trigeminus, fixando a localidade-tipo para Salvador - Bahia. Como é conhecida hoje, a espécie $O$. trigeminus apresenta ampla distribuição no Brasil, com registros para os estados de Pernambuco, Maranhão, Alagoas, Pará, Amapá, Bahia, Goiás, Minas Gerais, Rio de Janeiro, São Paulo, Mato Grosso do Sul e, também, na 
Guiana, ocorrendo apenas nas áreas de vegetação aberta (Cunha \& Nascimento, 1983; Zaher \& Caramaschi 1992).

Hoge \& Romano (1977) descreveram Oxyrhopus trigeminus guibei com base em dez espécimes procedentes dos estados do Paraná, São Paulo e Mato Grosso. O holótipo IBSP 27382 e grande parte dos parátipos foram perdidos no incêndio da Coleção Herpetológica do Instituto Butantan, ocorrido em 2010 em São Paulo, restando apenas um exemplar depositado na coleção do Museu de História Natural em Paris, França. Seus autores distinguiram a nova subespécie de $O$. trigeminus trigeminus por ela apresentar anéis pretos completos na parte posterior do corpo, maior número de ventrais e subcaudais e a ponta do focinho preta. Sendo que em O. t. trigeminus, o ventre é imaculado e o focinho é manchado de branco. Zaher \& Caramaschi (1992), apresentando ampla discussão entre os caracteres utilizados anteriormente e atualizando o status taxonômico destas espécies, elevaram Oxyrhopus guibei ao nível de espécie, descrevendo e utilizando como caracteres diagnósticos a morfologia hemipeniana, junto com os padrões de colorido e folidose. Oxyrhopus guibei Hoge \& Romano, 1977 é uma espécie abundante que ocorre em grande parte do Sudeste e Centro-Oeste do Brasil, incluindo áreas adjacentes do Paraná, Paraguai, Bolívia e Argentina (Hoge \& Romano, 1977; Zaher \& Caramaschi, 1992; Giraudo, 2001).

Os táxons aqui analisados não têm sido abordados em estudos com enfoque taxonômico, tampouco estão objetivamente diagnosticados. A falta de resolução na delimitação da distribuição geográfica de tais espécies, notoriamente parapátricas, somado à sobreposição em muitos dos caracteres de folidose, alimentam os problemas que persistem na definição de uma diagnose específica e geram frequentes confusões na determinação taxonômica destas espécies. 
Vale ressaltar que, por se tratar de serpentes "falsas-corais", o padrão de coloração e sua variação de cunho geográfico merecem atenção especial já que auxiliam de forma significativa na definição das possíveis unidades taxonômicas envolvidas nesse complexo de espécies com taxonomia confusa (Curcio, 2008; Bosque, 2012). Em um recente estudo, que associou o mimetismo ao padrão geográfico das espécies de Oxyrhopus e das corais-verdadeiras do gênero Micrurus, os autores afirmaram que devido à ampla distribuição geográfica e ecológica, ampla simpatria e grande variação nos padrões de coloração, Oxyrhopus representaria um excelente modelo para a investigação da evolução do mimetismo (Bosque et al, 2018). Portanto, compreender os padrões que estabelecem cada um dos táxons e suas distribuições geográficas, poderá auxiliar na classificação das espécies e inferir sobre possíveis padrões de distribuições baseados na história co-evolutiva entre gêneros filogeneticamente distantes. 


\section{CONCLUSÕES}

- A partir da análise de 929 exemplares são reconhecidas cinco espécies dentro do complexo de tríades do gênero Oxyrhopus;

- Oxyrhopus guibei - espécie plena que apresenta ampla distribuição geográfica em áreas de Mata Atlântica e enclaves de Cerrado. Possui em seu escopo uma população de ocorrência nos remanescentes de Mata Atlântica e nos brejos de altitude do nordeste que merece atenção especial e novos estudos com abordagens moleculares;

- Oxyrhopus trigeminus - espécie plena com extensa variação morfológica, que possui uma grande área de ocorrência nas regiões de Caatinga e Cerrado, ocorrendo também em áreas de enclave;

- Oxyrhopus vanidicus - espécie plena caracterizada por um padrão de coloração único dentre as espécies de tríades, e encontra-se restrita à Floresta Amazônica ao norte da bacia do rio Amazonas, que ainda carece de uma maior amostragem para elucidar a real distribuição da espécie;

- Oxyrhopus melanogenys - espécie plena que representa a unidade taxonômica M2, caracterizada por não apresentar tríades dispostas ao longo de todo o corpo e cauda;

- UTO G/M - trata-se de uma variação relacionada à O. melanogenys, na qual aparecem bandas ou tríades irregulares pelo corpo em pequeno número, muitas vezes anômalas. Não configura uma população estabelecida por critérios geográficos, sendo considerada apenas polimorfia da espécie em questão; 
- Oxyrhopus orientalis - a subespécie descrita anteriormente por Cunha \& Nascimento (1983) é elevada a nível de espécie plena caracterizada por apresentar tríades com as bandas externas mais estreitas que a banda central, dispostas ao longo de todo corpo e cauda, intercaladas com interespaços duas ou três vezes maiores que a largura das tríades. 


\section{RESUMO}

O gênero Oxyrhopus é composto por serpentes de porte médio, dentição opistóglifa, ovíparas, com pupilas elípticas e ampla distribuição neotropical. Nas espécies do gênero, há padrões dorsais de coloração uniformes, variegados, em bandas simples e/ou losangulares, mônades e tríades. O presente estudo traz uma revisão taxonômica do grupo de tríades pertencente ao gênero: $O$. guibei, $O$. melanogenys, $O$. trigeminus e $O$. vanidicus, visto que a semelhança morfológica no padrão de coloração e a sobreposição nos caracteres de folidose fundamentam problemas na definição da uma diagnose efetiva e geram frequentes confusões na determinação taxonômica destas espécies. Utilizando a análise de caracteres quantitativos (folidose e morfometria linear) e qualitativos da morfologia externa (coloração) e interna (hemipenis), foram analisados 1007 espécimes buscando representar e delimitar a distribuição geográfica de cada uma das espécies. Os exemplares foram agrupados em unidades taxonômicas operacionais, baseados nos caracteres de coloração, a fim de verificar a diversidade contida no grupo de espécies. As diferenças entre os agrupamentos foram avaliadas através de análises descritivas, univariadas e multivariadas. Os resultados permitiram reconhecer cinco espécies válidas, diagnosticáveis pelo padrão de coloração. A distribuição geográfica de cada espécie foi atualizada, bem como a variação merística e morfométrica. Não houveram mudanças de cunho taxonômico para $O$. guibei, O. trigeminus e $O$. vanidicus, entretanto, foram reconhecidas duas entidades taxonômicas em $O$. melanogenys. A população presente na porção ocidental da Amazônia representa o táxon descrito por Tschudi (1845) enquanto ao norte do Brasil (MA, MT e PA) a população anteriormente descrita como $O$. m. orientalis é elevada ao nível de espécie. 


\section{ABSTRACT}

The genus Oxyrhopus includes medium-size, opisthoglyphous and oviparous snakes, with elliptical pupils and wide distribution in the Neotropical region. The species of the genus have diferent dorsal color patterns: variegated, in simple and/or triangular bands, monads and triads. This study brings a taxonomic revision of the triad group: $O$. guibei, $O$. melanogenys, $O$. trigeminus and $O$. vanidicus. These species show morphological similarity in color pattern and overlap in many of folidose characters, which complicate the effective diagnosis and induce a frequent confusion in the taxonomic recognition of these species. The quantitative (scale count and linear morphometry) and qualitative characters of external (coloration) and internal (hemipenis) morphology were analyzed in 1007 specimens, seeking to cover the geographic range of each species. The specimens were grouped into operational taxonomic units, based primarily on qualitative color pattern, to verify the diversity contained in this group of species. The variation between groups was evaluated by descriptive, univariate and multivariate analyzes. Based on the results, this revision support the recognition of five full species, diagnosable by the color pattern. The geographical distribution of each species was updated, as well as meristic and morphometric variation. There were no taxonomic changes for Oxyrhopus guibei, O. trigeminus and O. vanidicus. However, two taxonomic entities were recognized in O. melanogenys. The population present in the western portion of Amazon forest represents the taxon described by Tschudi (1845), while in northern Brazil (Maranhão, Mato Grosso and abundantly in Pará) the population previously described as $O$. m. orientalis (Cunha \& Nascimento, 1983) is elevated to species rank. 


\section{REFERÊNCIAS BIBLIOGRÁFICAS}

ALENCAR, L. R. V.; GAIARSA, M. P.; MARTINS, M. The evolution of diet and microhabitat use in Pseudoboine snakes. South American Journal of Herpetology, v. 8, n. 1, p. 60-66, 2013.

ALENCAR, L. R. V.; GALDINO, C. A. B.; NASCIMENTO, L. B. Life history aspects of Oxyrhopus trigeminus (Serpentes: Dipsadidae) from two sites in southeastern Brazil. Journal of Herpetology, v. 46, n. 1, p. 9-14, 2012.

AMARAL, A. D. Notas sobre chromatismo de ophidios. I. Primeiro caso de erythrismo serpente, observado no Brasil. Memórias do Instituto Butantan, v. 7, p. 77-79, 1932.

AMARAL, A. Notas de Ophiologia: Da preferência do nome genérico Pseudoboa Schneider 1801 a Clelia Fitzinger, 1826 e Oxyrhopus Wagler, 1830. Revista do Museu Paulista, v.14, p. 10-16, 1926.

ARGOLO, A. J. S. As serpentes dos caucais do sudeste da Bahia. Ilhéus - Bahia, Editus, 260p, 2004.

BAILEY, J. R. Oxyrhopus. In: PETERS, J.A.; OREJAS-MIRANDA B. (Org.).

Catalogue of the Neotropical Squamata. Part I: Snakes. Washington: Smithsonian Institution Press, 1970. p. 229-235.

BAILEY, J. R. The synthetic approach to Colubrid classification. Herpetologica, v. 23, n. 2, p. 155-161, 1967.

BERNARDO, P. H. Revisão taxonômica e sistemática de Oxyrhopus clathratus Duméril, Bibron \& Duméril, 1854 (Serpentes, Dispsadidade; Pseudoboini). Dissertação de Mestrado, Universidade Estadual Paulista, campus Rio Claro, São Paulo, 2010.

BITAR, Y. O. C. \& SANTOS-COSTA, M. C. Biologia reprodutiva e alimentar de Oxyrhopus melanogenys Tschudi, 1845, na Amazônia Oriental. Belém, Pará, Universidade Federal do Pará. (Relatório Técnico Científico. PIBIC/UFPA), 2006.

BOSQUE, R. J. Mimetismo, padrões de coloração e distribuição geográfica de serpentes Oxyrhopus Wagler, 1830 (Colubridae) e Micrurus Wagler, 1824 (Elapidae), 2012. 
BOSQUE, R. J.; LAWRENCE, J. P.; BUCHHOLZ, R.; COLLI, G. R.; HEPPARD, J. \& NOONAN, B. Diversity of warning signal and social interaction influences the evolution of imperfect mimicry. Ecology and Evolution, v. 8, n. 15, 7490-7499, 2018.

BOSQUE, R. J.; LAWRENCE, J. P.; BUCHHOLZ, R.; COLLI, G.; HEPPARD, J.;

NOONAN, B. Diversity of warning signal and social interaction influences the evolution of imperfect mimicry. Ecology and evolution, v. 8, n. 15, p. 7490-7499, 2018.

BOULENGER, G. A. Catalogue of the snakes in British Museum (Natural History)

3. London: British Museum, 727p, 1896.

BRANDÃO, A. L. P. Posição taxonômica de Oxyrhopus aff. guibei e variações dos padrões de Oxyrhopus melanogenys (Tschudi, 1845) (Serpentes: Dipsadidae). Dissertação apresentada ao programa de Pós-Graduação em Ciências Biológicas (Zoologia), Museu Nacional, da Universidade Federal do Rio de Janeiro, 2013.

CADLE, J. E. Molecular Systematics of Neotropical Xenodontine Snakes: I. South American Xenodontines. Herpetologica, v. 40, n. 1, p. 8-20, 1984.

CAMPBELL, J. A. \& LAMAR, W. W. The venemous reptiles of Latin America, Comstock, Cornell University Press, Ithaca and New York, 425p, 1989.

CAMPBELL, J. A. \& LAMAR, W. W. The venomous reptiles of the western hemisphere. Comstock, Cornell University Press, 976p, 2004.

CUNHA, O. R. \& NASCIMENTO, F. P. de. Ofidios da Amazônia. X - As cobras da região leste do Pará. Belém, Museu Paraense Emílio Goeldl, 218 p, 1978.

CUNHA, O. R.; NASCIMENTO, F. P. Ofídios da Amazônia: As espécies de Oxyrhopus Wagler, com uma subespécie nova, e Pseudoboa Schneider, na Amazônia oriental e Maranhão (Ophidia, Colubridae). Boletim do Museu Paraense Emílio Goeldi, v. 122, n. 42, p. 1-47, 1983.

CURCIO, F. F. Revisão taxonômica e variação geográfica do gênero Erythrolamprus Boie (Serpentes, Xenodontinae). Vol. I: Tese de Doutorado, Universidade do São Paulo, 2008.

DIXON, J. R. \& SOINI, P. The reptiles of the upper Amazon basin, Iquitos region, Peru. II. Crocodilians, turtles and snakes. Contribution in Biology and Geology, Milwaukee Public Museum, p. 1-71, 1977. 
DIXON, J. R. \& SOINI, P. The Reptiles of the Upper Amazon Basin, Iquitos Region, Peru. Part I. Lizards and Amphisbaenians. Part II. Crocodilians, Turtles and Snakes. Milwaukee Public Museum, 154 p, 1986.

DOWLING, H. G. A proposed standart system of counting ventral in snakes. British Journal of Herpetology, 1: 97-99, 1951.

DUELLMAN, W. E. The biology of an equatorial herpetofauna in Amazonian. Ecuador. Lawrence: University of Kansas, 352 p, 1978.

DUNN, E. R. Los Géneros de Anfibios y reptiles de Colombia, III: Tercera Parte: Reptiles; Orden de las Serpientes. Caldasia, 3(12), p. 155-224, 1944.

FERRAREZZI, H. \& FREIRE, E. M. X. New species of Bothrops Wagler, 1824 from the Atlantic forest of northeastern Brazil (Serpentes, Viperidae, Crotalinae). Boletim do Museu Nacional, Nova Serie, Zoologia, v. 440, p. 1-10, 2001.

FIGUEROA, A.; MCKELVY, A. D.; GRISMER, L. L.; BELL, C. D.; LAILVAUX, S. P. A species-level phylogeny of extant snakes with description of a new colubrid subfamily and genus. PLoS One. 2016; 11: e0161070. https://doi.org/10.1371/journal.pone.0161070 PMID: 27603205.

FREIRE, E. M .X., CARAMASCHI, U. \& ARGOLO, A. J. S. A new species of Liotyphlops (Serpentes: Anomalepididae) from the Atlantic Rain Forest of Northeastern Brazil. Zootaxa, v. 1393, p. 19-26, 2007.

FREIRE, E. M. X.; CARAMASCHI, U.; GONÇALVES, U. A new species of Dendrophidion (Serpentes: Colubridae) from the PIRES ET AL. 582 - Zootaxa 3811 (4) (C) 2014 Magnolia Press Atlantic Rain Forest of Northeastern Brazil. Zootaxa, v. 2719, p. 62-68, 2010.

FREITAS, M. A.; SILVA, T. F. S. A herpetofauna das caatingas e áreas de altitudes do nordeste brasileiro. Pelotas, USEB, 388 p., 2007.

GAIARSA, M. P.; ALENCAR, L. R. V.; MARTINS, M. Natural history of Pseudoboine snakes. Papéis Avulsos de Zoologia (São Paulo), v. 53, n. 19, p. 261-283, 2013.

GIRAUDO, A. Serpientes de la Selva Paranaense y del Chaco Húmedo. Literature of Latin America Ed., Buenos Aires, 325 p., 2001.

GOMES, J. F. Contribuição para o conhecimento dos ophidios do Brasil. II. Ophidios do Museu Rocha (Ceará). Revista do Museu Paulista, v. 10, p. 505-527, 1918. 
GRAZZIOTIN, F. G.; ZAHER, H.; MURPHY, R. W.; SCROCCHI, G.; BENAVIDES, M. A.; ZHANG, Y. P. \& BONATTO, S. L. Molecular phylogeny of the new world Dipsadidae (Serpentes: Colubroidea): a reappraisal. Cladistics, 28(5), p. 437-459, 2012.

GÜNTHER, A. Seventh account of new species of snakes in the collection of the British Museum. Ann. Mag. Nat. Hist. (4) 9: p. 13-37, 1872.

HE, M.; FENG, J.; LIU, S.; GUO, P.; ZHAO, E. The phylogenetic position of Thermophis (Serpentes: Colubridae), an endemic snake from the Qinghai-Xizang Plateau, China. J Nat Hist, v. 43: p. 479-488, 2009.

HOGE, A. R. \& ROMANO, S. A. R. W. L. Description of a new subsp. of Oxyrhopus Wagler. [Serpentes: Colubridae]. Mem. Int. Butantan, v. 40, n. 41, p. 55-62, 1977. HOGE, A. R.; SANTOS, N. P.; LOPES, L. A. \& SOUZA, I. M. Serpentes coletadas pelo projeto Rondon VII em Iauaretê, Brasil. Memorias Instituto Butatan, v. 36, p. 221-232, 1973 [1972].

JENNER, J. V.; DOWLING, H. G. Taxonomy of american xenodontine snakes. Herpetologica, v. 41, n. 2, p. 161-172, 1985.

KORNACKER, P. M. Checklist and key to the snakes of Venezuela. Germany, ed Pako-Verlag,1 edition, p. 119-121, 1999.

LYNCH, J. D. Snakes of the Genus Oxyrhopus (Colubridae: Squamata) in Colombia: Taxonomy and Geographic Variation. Papéis Avulsos de Zoologia, v. 49, n.25, p. 319-337, 2009.

MARTINS, M; OLIVEIRA, M. E. Natural history of snakes in forests of the Manaus region, Central Amazonia, Brazil. Herpetological Natural History, v. 6, n. 2, p. 78-150, 1998.

MCCULlOCH, R. D.; LATHROP, A.; KOK, P. J. R.; ERNST, R.; KALAMANDEEN, M. The genus Oxyrhopus (Serpentes: Dipsadidae: Xenodontinae) in Guyana: morphology, distributions and comments on taxonomy. Papéis Avulsos de Zoologia, v. 49, n. 36, p. 487-495, 2009.

OGUIURA, N.; FERRAREZZI, H.; BATISTIC, R. F. Cytogenetics and molecular data in snakes: a phylogenetic approach. Cytogenetic and genome research, v. 127, n. 2-4, p. 128-142, 2009. 
PASSOS, P.; FERNANDES, R.; BERNILS, R.S.; MOURA-LEITE, J.C. Taxonomic revision of the Brazilian Atlantic Forest Atractus (Reptilia: Serpentes: Dipsadidae). Zootaxa, 2364, p. 1-63, 2010.

PEMA. Plano de Manejo do Parque Estadual Monte Alegre. MMA/Secretaria de Extrativismo e Desenvolvimento Rural Sustentável, PROECOTUR, SEMA/PA, 2009.

PEREZ SANTOS, C. \& MORENO, A. G. Ofidios de Colombia. Museo Regionale di Scienze Naturali di Torino, 517 p., 1988.

PEREZ-SANTOS, C.; MORENO, A. G. Ofídios de Colômbia. Museo Regionale de Scienze Naturali Torino, Madrid, p. 257-265, 1988.

PIRES, M. G.; DA SILVA J. R. N. J.; FEITOSA, D. T.; PRUDENTE FILHO, A. L. A new species of triadal coral snake of the genus Micrurus Wagler, 1824 (Serpentes: Elapidae) from northeastern Brazil. Zootaxa, v. 3811(4), p. 569-584, 2014.

PIZZATTO, L.; MARQUES, O. A. V. Reproductive biology of the false coral snake Oxyrhopus guibei (Colubridae) from Southeastern Brazil. Amphibia-Reptilia, v. 23, n. 4, p. 495-504, 2002.

PYRON, R. A.; BURBRINK, F. T.; COLLI, G. R.; MONTES DE OCA, A. N.; VITT, L. J.; KUCZYNSKI, C. A. The phylogeny of advanced snakes (Colubroidea), with discovery of a new subfamily and comparison of support methods for likelihood trees. Mol Phylogenet Evol., v. 58, p. 329-342, 2011. https://doi.org/10.1016/j.ympev. 2010.11.006 PMID: 21074626

PYRON, R. A.; BURBRINK, F. T.; WIENS, J. J. A phylogeny and revised classification of Squamata, including 4161 species of lizards and snakes. BMC Evol. Biol., v. 13, n. 93, 2013. https://doi.org/10.1186/1471-2148-13-93 PMID: 23627680.

R DEVELOPMENT CORE TEAM. R: A language and environment for statistical computing. R Foundation for Statistical Computing, Vienna, Austria. ISBN 3900051-07-0, URL. Disponível em: http://www.R-project.org, 2009.

ROZE, J. A. La taxonomı y zoogeografia de los ofidios en Venezuela. Caracas: Universidad Central de Venezuela, 1966. 
SÁNCHEZ-C, H.; CASTAÑO-M., O. V. \& CÁRDENAS-A., G. Diversidad de los reptiles en Colombia. In: Rangel Ch., J.O. (Ed.), Colombia Diversidad Biotica I. Universidad Nacional de Colombia, Bogotá, Colombia, p. 277-325, 1995.

SHINE, R. Ecological causes for the evolution of sexual dimorphism: a review of the evidence. The Quarterly Review of Biology, v. 64, n. 4, p. 419-461, 1989.

SHINE, R. Sexual dimorphism. In: Snakes: the ecology and behavior. (Eds) R. Seigel \& J. Collins, New York. McGraw-Hill, 1993.

SILVA-JR., N.J. The snakes from Samuel hydroelectric power plant and vicinity, Rondônia, Brasil. Herpetological Natural History, v. 1, p. 37-86, 1993.

STEJNEGER, L. An annotated list of batrachians and reptiles collected in the vicinity of La Guaira, Venezuela, with descriptions of two new species of snakes. Proceedings of the United States National Museum, v. 24, n. 1901, p. 179-202, 1901.

TSCHUDI, J. J. Reptilium conspectum quae in republica Peruana reperiuntur er pleraque observata vel collecta sunt in itenere. Archiv für Naturgeschichte, v. 11, n. 1, p. $150-170,1845$.

TSCHUDI, J. J. Untersuchungen uber die Fauna Peruana. Herpetologie. St. Gallen, p. 49, pl. 4, 1846.

UETZ, P.; FREED, P.; HOŠEK, J. The Reptile Database, Disponível em: http://www.reptile-database.org. Acessado em novembro de 2019.

VANZOLINI, P. E. Notas sobre os ofídios e lagartos da Cachoeira de Emas, no município de Pirassununga, Estado de São Paulo. Revista Brasileira de Biologia, v. 8 , n. 3, p. 377-400, 1948.

VANZOLINI, P. E.; RAMOS-COSTA, A. M. M.; VITT, L. J. Répteis da Caatinga. Academia Brasileira de Ciências, Rio de Janeiro, 161 p., 1980.

VIDAL, N.; DEWYNTER, M.; GOWER, D. Dissecting the major American snake radiation: A molecular phylogeny of the Dipsadidae Bonaparte (Serpentes, Caenophidia). Comptes Rendus Biologies v. 333, n. 2010, p. 48-55, 2010.

VIDAL, N.; KINDL, S. G.; WONG, A.; HEDGES, S. B. Phylogenetic relationships of xenodontine snakes inferred from $12 \mathrm{~S}$ and $16 \mathrm{~S}$ ribosomal RNA sequences. Molecular Phylogenetics and Evolution, v. 14, n. 3, p. 389-402, 2000.

ZAHER, H. Hemipenial morphology of the south american xenodontine snakes, with a proposal for a monophyletic Xenodontinae and a reappraisal of Colubroid 
hemipenis. Bulletin of the American Museum of Natural History, v. 240, p. 168, 1999.

ZAHER, H. Phylogénie des Pseudoboini et évolution des Xenodontinae sud américans (Serpentes, Colubridae). Tese (Doutorado) - Muséum National d'histoire Naturelle, Paris, 1994.

ZAHER, H.; CARAMASCHI, U. Sur le statut taxonomique d' Oxyrhopus trigeminus et $O$. guibei (Serpentes, Xenodontinae). Bulletin de Muséum National d'histoire Naturelle (ser. 4), v. 14A, n. 3-4, p. 805-827, 1992.

ZAHER, H.; GRAZZIOTIN, F.G.; CADLE, J.E.; MURPHY, R.W.; MOURALEITE, J.C.; BONATTO, S. Molecular Phylogeny of advanced snakes (Serpentes, Caenophidia) with an emphasis on South American Xenodontines: A revised classification and descriptions of new taxa. Papéis Avulsos de Zoologia, v. 49, n. 11, p. 115-153, 2009.

ZAHER, H.; MURPHY, R. W.; ARREDONDO, J. C.; GRABOSKI, R.; MACHADOFILHO, P. R.; MAHLOW, K.; ... \& ZHANG, Y. P. Large-scale molecular phylogeny, morphology, divergence-time estimation, and the fossil record of advanced caenophidian snakes (Squamata: Serpentes). PloS one, v. 14, n. 5, 2019.

ZAHER, H.; PRUDENTE, A. L. C. Hemipenes of Siphlophis (Serpentes, Xenodontinae) and techniques of hemipenial preparation in snakes: a response to Dowling. Herpetological Review, v. 34, n. 4, p. 302, 2003.

ZAHER, H.; PRUDENTE, A. L. C. Intraespecific Variation of the Hemipenis in Siphlophis and Tripanurgos. Journal of Herpetology, v. 33, n. 4, p. 698-702, 1999. 